PROCEEDINGS OF THE

AMERICAN MATHEMATICAL SOCIETY

Volume 126, Number 8, August 1998, Pages 2407-2416

S 0002-9939(98)04603-6

\title{
LAPLACE TRANSFORMS AND GENERATORS OF SEMIGROUPS OF OPERATORS
}

\author{
JIGEN PENG AND SI-KIT CHUNG
}

(Communicated by Palle E. T. Jorgensen)

\begin{abstract}
In this paper, a characterization for continuous functions on $(0, \infty)$ to be the Laplace transforms of $f \in L^{\infty}(0, \infty)$ is obtained. It is also shown that the vector-valued version of this characterization holds if and only if the underlying Banach space has the Radon-Nikodým property. Using these characterizations, some results, different from that of the Hille-Yosida theorem, on generators of semigroups of operators are obtained.
\end{abstract}

\section{INTRODUCTION}

The theory of Laplace transforms plays an important role in the theory of semigroups of operators. Given a function $F$ on $(0, \infty)$, under what conditions is $F$ the Laplace transform of a certain function $f$ ? This problem has been investigated extensively. In [7], Widder obtained the following characterization of Laplace transforms of scalar-valued functions:

A function $F$ on $(0, \infty)$ is the Laplace transform of $f \in L^{\infty}(0, \infty)$ if and only if $F$ is infinitely differentiable and satisfies

$$
\sup \left\{\left|\frac{1}{n !} \lambda^{n+1} F^{(n)}(\lambda)\right|: \lambda>0, n \in \mathbf{N} \cup\{0\}\right\}<\infty .
$$

The vector-valued version of Widder's theorem has been investigated by Arendt among others. In [1], Arendt obtained an "integrated version of Widder's theorem" (see [1, Theorem 1.1]), and from this generalization, the relation between the HilleYosida theorem and Widder's theorem is revealed.

It is worth noting that in Widder's characterization of Laplace transforms, condition $\left(\mathrm{W}_{\infty}\right)$ involves not only the original function, but also its higher derivatives, and so in certain practical problems it may be difficult to verify condition $\left(\mathrm{W}_{\infty}\right)$. In Section 2, we give a characterization of Laplace transforms which involves only the original function but not its derivatives. Applications of this characterization can be found in [6].

In the theory of semigroups of operators, it is known that whether a linear operator $A$ is the generator of a certain semigroup $\left(C_{0}\right.$-semigroup or integrated semigroup) is related to the Laplace representation of its resolvent $R(\lambda, A)$ (see [1], [5], [3]). In Section 3, using the results in Section 2, we obtain some characterization

Received by the editors March 18, 1996 and, in revised form, January 23, 1997.

1991 Mathematics Subject Classification. Primary 47D03; Secondary 44A10.

(C)1998 American Mathematical Society 
results for generators of semigroups of operators. These results are different from those given by the Hille-Yosida theorem.

\section{Characterizations of Laplace transforms}

Let $f \in L^{\infty}(0, \infty)$. The Laplace transform $F$ of $f$ is given by

$$
F(\lambda)=\int_{0}^{\infty} e^{-\lambda t} f(t) d t \quad(\lambda>0) .
$$

The following result gives a characterization of those $F \in C(0, \infty)$ that are Laplace transform of an element $f$ in $L^{\infty}(0, \infty)$. This characterization involves only the original function $F$, not its higher derivatives.

Theorem 2.1. Let $F \in C(0, \infty)$. The following assertions are equivalent.

1. $F$ is the Laplace transform of some $f \in L^{\infty}(0, \infty)$.

2. There exists a constant $M$ such that $|\lambda F(\lambda)| \leq M$ for a.e. $\lambda>0$ and $\left|\sum_{j=1}^{\infty} \frac{(-1)^{j-1}}{(j-1) !} e^{j n} \lambda F(j \lambda)\right| \leq M$ for a.e. $\lambda>0$ for infinitely many $n \in \mathbf{N}$.

3. Same as (2), with the inequalities holding for all $\lambda>0$ and all $n \in \mathbf{N}$.

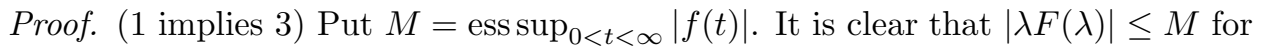
all $\lambda>0$. Let $\lambda>0$ and $n \in \mathbf{N}$. Then

$$
\begin{aligned}
\left|\sum_{j=1}^{\infty} \frac{(-1)^{j-1}}{(j-1) !} e^{j n} \lambda F(j \lambda)\right| & =\left|\int_{0}^{\infty} \lambda \sum_{j=1}^{\infty} \frac{(-1)^{j-1}}{(j-1) !} e^{j n} e^{-j \lambda t} f(t) d t\right| \\
& =\left|\int_{0}^{\infty} \lambda e^{-e^{n-\lambda t}} e^{n-\lambda t} f(t) d t\right| \\
& \leq M .
\end{aligned}
$$

(3 implies 2) Obvious.

(2 implies 1) Let $f_{n}(t)=\sum_{j=1}^{\infty} \frac{(-1)^{j-1}}{(j-1) !} e^{j n} \frac{n}{t} F\left(\frac{j n}{t}\right)$. Then the given condition on $F$ implies that there exist $n_{1}<n_{2}<\cdots$ such that $\left(f_{n_{i}}\right)$ is a bounded sequence in $L^{\infty}(0, \infty)$. Since $L^{\infty}(0, \infty)$ is the dual of the separable space $L^{1}(0, \infty),\left(f_{n_{i}}\right)$ has a subsequence $\left(f_{n_{i_{k}}}\right)$ which converges in the weak*-topology to $f \in L^{\infty}(0, \infty)$. In particular, for every $\lambda>0$,

$$
\lim _{k \rightarrow \infty} \int_{0}^{\infty} e^{-\lambda t} f_{n_{i_{k}}}(t) d t=\int_{0}^{\infty} e^{-\lambda t} f(t) d t
$$

On the other hand, since

$$
\int_{0}^{\infty} \sum_{j=1}^{\infty} \frac{e^{j n}}{(j-1) !} \frac{n}{t}\left|F\left(\frac{j n}{t}\right)\right| e^{-\lambda t} d t<\infty
$$

and

$$
\int_{0}^{\infty} \sum_{j=1}^{\infty} \frac{e^{j n}}{(j-1) !} \frac{n}{s}\left|F\left(\frac{1}{s}\right)\right| e^{-\lambda j n s} d s<\infty
$$

we have

$$
\begin{aligned}
\int_{0}^{\infty} f_{n}(t) e^{-\lambda t} d t & =\int_{0}^{\infty} \sum_{j=1}^{\infty} \frac{(-1)^{j-1}}{(j-1) !} e^{j n} \frac{n}{t} F\left(\frac{j n}{t}\right) e^{-\lambda t} d t \\
& =\sum_{j=1}^{\infty} \frac{(-1)^{j-1}}{(j-1) !} e^{j n} \int_{0}^{\infty} \frac{n}{s} F\left(\frac{1}{s}\right) e^{-\lambda j n s} d s
\end{aligned}
$$




$$
\begin{aligned}
& =\int_{0}^{\infty} e^{-e^{n(1-\lambda s)}} e^{n(1-\lambda s)} \frac{n}{s} F\left(\frac{1}{s}\right) d s \\
& =\int_{-n}^{\infty} e^{-e^{-u}} e^{-u} \frac{n}{n+u} F\left(\frac{\lambda n}{n+u}\right) d u \\
& =\int_{-\infty}^{\infty} \chi_{(-n, \infty)} e^{-e^{-u}} e^{-u} \frac{n}{n+u} F\left(\frac{\lambda n}{n+u}\right) d u,
\end{aligned}
$$

so by the dominated convergence theorem (using the condition that $|\lambda F(\lambda)| \leq M$ a.e. $\lambda>0)$,

$$
\lim _{n \rightarrow \infty} \int_{0}^{\infty} f_{n}(t) e^{-\lambda t} d t=\int_{-\infty}^{\infty} e^{-e^{-u}} e^{-u} F(\lambda) d u=F(\lambda) .
$$

Hence $F$ is the Laplace transform of $f$.

In the proof of the above theorem, we use the following version of the dominated convergence theorem: if $\int_{X} \sum_{j=1}^{\infty}\left|g_{j}\right|<\infty$, then $\int_{X} \sum_{j=1}^{\infty} g_{j}=\sum_{j=1}^{\infty} \int_{X} g_{j}$. This kind of argument will be used in later proofs and will not be mentioned explicitly.

Corollary 2.2. Suppose a continuous function $F$ on $(0, \infty)$ satisfies

$$
\sup _{\lambda>0}|\lambda F(\lambda)|<\infty
$$

and

$$
\sup _{\lambda>0, n \in \mathbf{N}}\left|\sum_{j=1}^{\infty} \frac{(-1)^{j-1}}{(j-1) !} e^{j n} \lambda F(j \lambda)\right|<\infty .
$$

Then $F$ is infinitely differentiable and can be extended to an analytic function on the right half-plane $\{z \in \mathbf{C}: \operatorname{Re} z>0\}$.

Note that unlike Bernstein's theorem on completely monotone functions (see [7]), the condition given in the above corollary does not involve higher derivatives of $F$.

Next we want to consider Laplace transforms of vector-valued functions. Given $f \in L^{\infty}((0, \infty), E)$, where $E$ is a Banach space, using the same argument as in the proof of Theorem 2.1, we see that the Laplace transform $F$ of $f$ satisfies

$$
\left(\mathrm{P}_{\infty}\right) \quad \sup _{\lambda>0}\|\lambda F(\lambda)\|<\infty \quad \text { and } \quad \sup _{\lambda>0, n \in \mathbf{N}}\left\|\sum_{j=1}^{\infty} \frac{(-1)^{j-1}}{(j-1) !} e^{j n} \lambda F(j \lambda)\right\|<\infty .
$$

We will show that the converse holds if $E$ has the Radon-Nikodým property. In fact, this gives a characterization for Banach spaces with the Radon-Nikodým property. The idea is to show that condition $\left(\mathrm{P}_{\infty}\right)$ is equivalent to Widder's condition.

Theorem 2.3. Let $E$ be a Banach space and let $F \in C((0, \infty), E)$. The following assertions are equivalent.

1. There exists a Lipschitz continuous function $\alpha:[0, \infty) \longrightarrow E$ with $\alpha(0)=0$ such that

$$
F(\lambda)=\int_{0}^{\infty} \lambda e^{-\lambda t} \alpha(t) d t \quad \forall \lambda>0 .
$$

2. F satisfies condition $\left(\mathrm{P}_{\infty}\right)$.

3. $F$ is infinitely differentiable and $\sup \left\{\left\|\frac{1}{n !} \lambda^{n+1} F^{(n)}(\lambda)\right\|: \lambda>0, n \in \mathbf{N} \cup\{0\}\right\}<$ $\infty$. 
Proof. (1 implies 2) Let $x^{*} \in E^{*}$. Consider the scalar-valued function $g(t)=$ $\left\langle\alpha(t), x^{*}\right\rangle$. The conditions on $\alpha$ imply that there exists $f \in L^{\infty}(0, \infty)$ such that $g(t)=\int_{0}^{t} f(s) d s$ for all $t \geq 0$. So for every $\lambda>0$, we have (using Fubini's theorem)

$$
\left\langle F(\lambda), x^{*}\right\rangle=\int_{0}^{\infty}\left(\lambda e^{-\lambda t} \int_{0}^{t} f(s) d s\right) d t=\int_{0}^{\infty} e^{-\lambda t} f(t) d t
$$

Using the proof of Theorem 2.1 together with the uniform boundedness principle, we see that $F$ satisfies condition $\left(\mathrm{P}_{\infty}\right)$.

(2 implies 1) For every $x^{*} \in E^{*}$, we consider the function $\lambda \mapsto\left\langle F(\lambda), x^{*}\right\rangle$. By Theorem 2.1, there exists $\tilde{f}_{x^{*}} \in L^{\infty}(0, \infty)$ such that

$$
\left\langle F(\lambda), x^{*}\right\rangle=\int_{0}^{\infty} e^{-\lambda t} \tilde{f}_{x^{*}}(t) d t \quad \forall \lambda>0 .
$$

It follows from the proof of [1, Theorem 1.1] that there exists a function $\alpha$ which satisfies the requirements.

The equivalence of 1 and 3 is just [1, Theorem 1.1].

Theorem 2.4. A Banach space $E$ has the Radon-Nikodym property if and only if every $F \in C((0, \infty), E)$ satisfying condition $\left(\mathrm{P}_{\infty}\right)$ is the Laplace transform of some $f \in L^{\infty}((0, \infty), E)$.

Proof. This is an immediate consequence of Theorem 2.3 and [1, Theorem 1.4].

Remark 2.1. If $E$ is a dual space and has the Radon-Nikodým property, then $L^{\infty}((0, \infty), E)$ is a dual space (see [4]). So given $F \in C((0, \infty), E)$ satisfying condition $\left(\mathrm{P}_{\infty}\right)$, the bounded sequence $\left(f_{n}\right)$ constructed in the proof of Theorem 2.1 has a weak* limit $f$ which is the inverse Laplace transform of $F$.

For continuous $f \in L^{\infty}((0, \infty), E)$, where $E$ is a Banach space not necessarily possessing the Radon-Nikodým property, we have the following inversion formula.

Theorem 2.5. Let $E$ be a Banach space. Let $f:(0, \infty) \longrightarrow E$ be a bounded continuous function and $F$ its Laplace transform. Then

$$
f(t)=\lim _{n \rightarrow \infty} \sum_{j=1}^{\infty} \frac{(-1)^{j-1}}{(j-1) !} e^{j n t} n F(j n) \quad \forall t>0,
$$

the convergence is uniform on compact subsets of $(0, \infty)$, and uniform on bounded subsets of $(0, \infty)$ if $f(0+)=\lim _{t \rightarrow 0+} f(t)$ exists, and in this case,

$$
f(0+)=\left(1-e^{-1}\right)^{-1} \lim _{n \rightarrow \infty} n \sum_{j=1}^{\infty} \frac{(-1)^{j-1}}{(j-1) !} F(j n) .
$$

Proof. Let $t \geq 0$ and $n \in \mathbf{N}$. Then

$$
\begin{aligned}
\lim _{n \rightarrow \infty} & \sum_{j=1}^{\infty} \frac{(-1)^{j-1}}{(j-1) !} e^{j n t} n F(j n)=\lim _{n \rightarrow \infty} \int_{0}^{\infty} n \sum_{j=1}^{\infty} \frac{(-1)^{j-1}}{(j-1) !} e^{j n t} e^{-j n r} f(r) d r \\
& =\lim _{n \rightarrow \infty} \int_{0}^{\infty} n e^{-e^{n(t-r)}} e^{n(t-r)} f(r) d r \\
& =\lim _{n \rightarrow \infty} \int_{-n t}^{\infty} e^{-e^{-u}} e^{-u} f\left(\frac{n t+u}{n}\right) d u
\end{aligned}
$$




$$
= \begin{cases}\int_{-\infty}^{\infty} e^{-e^{-u}} e^{-u} f(t) d u & \text { if } t>0 \\ \int_{0}^{\infty} e^{-e^{-u}} e^{-u} f(0+) d u & \text { if } t=0 \text { and } f(0+) \text { exists }\end{cases}
$$

where the last equality follows from the dominated convergence theorem and the condition that $f$ is continuous. Since $f$ is uniformly continuous on $[a, b]$ for $0<$ $a<b<\infty$ (on $(0, b]$ if $f(0+)$ exists), the convergence given in the last equality is uniform on $[a, b]$ (on $(0, b]$ if $f(0+)$ exists).

Remark 2.2. Using the same idea as in the above proof, we see that the sequence $\left(f_{n}\right)$ constructed in the proof of Theorem 2.1 converges to $f$ for all $t>0$ if $f$ is continuous. However, we cannot consider the convergence at $t=0$ for this sequence.

\section{SEMigroups of OPERATORS}

Let $E$ be a Banach space. The space of all bounded linear operators from $E$ into itself is denoted by $\mathcal{B}(E)$. A family $(S(t))_{t>0} \subset \mathcal{B}(E)$ is said to be a semigroup if $S(s+t)=S(s) S(t)$ for all $s, t>0$. If $(S(t))_{t>0}$ is a strongly continuous semigroup and SOT- $\lim _{t \rightarrow 0+} S(t)=I:=S(0),(S(t))_{t \geq 0}$ is called a $C_{0}$-semigroup.

Proposition 3.1. Let $E$ be a Banach space. Let $A: \mathcal{D}(A) \subset E \longrightarrow E$ be a closed linear operator and let $w \in \mathbf{R}$. If there exists a strongly continuous semigroup $(S(t))_{t>0} \subset \mathcal{B}(E)$ satisfying $\|S(t)\| \leq M e^{w t}$ for all $t>0$, where $M$ is a constant, such that for all $x \in E$,

$$
R(\lambda, A) x=\int_{0}^{\infty} e^{-\lambda t} S(t) x d t \quad \forall \lambda>w,
$$

then $(w, \infty) \subset \rho(A)$ and the function $F:(0, \infty) \longrightarrow \mathcal{B}(E)$ defined by

$$
F(\lambda)=R(w+\lambda, A)
$$

satisfies condition $\left(\mathrm{P}_{\infty}\right)$. The converse is true if $E$ has the Radon-Nikodým property.

Proof. The condition on $(S(t))_{t>0}$ implies that $F$ is the Laplace transform (in the strong operator topology) of the bounded function $t \mapsto e^{-w t} S(t)$. Hence $F$ satisfies condition $\left(\mathrm{P}_{\infty}\right)$.

Conversely, if $F$ satisfies condition $\left(\mathrm{P}_{\infty}\right)$, by Theorem 2.3, it satisfies the HilleYosida condition, namely,

$$
\sup _{\lambda>0, m \in \mathbf{N} \cup\{0\}}\left\|(\lambda R(\lambda, A-w))^{m}\right\|<\infty .
$$

Hence by [1, Theorem 6.2], there exists a strongly continuous semigroup $(T(t))_{t>0}$ satisfying $\sup _{t>0}\|T(t)\|<\infty$ such that $R(\lambda, A-w) x=\int_{0}^{\infty} e^{-\lambda t} T(t) x d t$ for all $\lambda>0, x \in E$. Hence $\left(S(t)=e^{w t} T(t)\right)_{t>0}$ is the required semigroup.

Remark 3.1. The converse is also true if $A$ is densely defined. In this case, the strongly continuous semigroup $(S(t))_{t>0}$ can be extended to a $C_{0}$ semigroup $(S(t))_{t \geq 0}$ (see Corollary 3.7). 
Proposition 3.2. Let $E$ be a Banach space. Let $w \in \mathbf{R}$. Suppose $A: \mathcal{D}(A) \subset$ $E \longrightarrow E$ is the generator of a $C_{0}$-semigroup $(S(t))_{t \geq 0}$ with $\|S(t)\| \leq M e^{w t}$ for all $t \geq 0$, where $M$ is a constant. Then for every $x \in E$, we have

$$
\begin{aligned}
S(t) x & =e^{w t} \lim _{n \rightarrow \infty} n \sum_{j=1}^{\infty} \frac{(-1)^{j-1}}{(j-1) !} e^{j n t} R(j n+w, A) x \quad \text { for } t>0, \\
\left(1-e^{-1}\right) x & =\lim _{n \rightarrow \infty} n \sum_{j=1}^{\infty} \frac{(-1)^{j-1}}{(j-1) !} R(j n+w, A) x,
\end{aligned}
$$

and the convergence is uniform on $(0, b]$ for $0<b<\infty$.

Proof. This is an immediate consequence of Theorem 2.5

Let $n \in \mathbf{N}$. A strongly continuous family $(S(t))_{t \geq 0} \subset \mathcal{B}(E)$ is called an $n$-times integrated semigroup if $S(0)=0$ and, for all $x \in E$,

$$
S(t) S(s) x=\frac{1}{(n-1) !}\left[\int_{t}^{s+t}(s+t-r)^{n-1} S(r) x d r-\int_{0}^{s}(s+t-r)^{n-1} S(r) x d r\right]
$$

$\forall s, t \geq 0$. For convenience, a $C_{0}$-semigroup is also called a 0 -times integrated semigroup.

An $n$-times integrated semigroup $(S(t))_{t \geq 0}$ (where $n \in \mathbf{N}$ ) is said to be

1. exponentially bounded if there exist constants $M$, w such that $\|S(t)\| \leq M e^{w t}$ for all $t \geq 0$;

2. non-degenerate if $S(t) x=0$ for all $t \geq 0$ implies $x=0$;

3. locally Lipschitz if there exist constants $M, w$ such that $\|S(t+h)-S(t)\| \leq$ $M e^{w(t+h)} h$ for all $t, h \geq 0$.

Given a non-degenerate, exponentially bounded $n$-times integrated semigroup $(S(t))_{t \geq 0}$ (where $n \in \mathbf{N}$ ), there exists a unique operator $A$ and there exists $a \in \mathbf{R}$ with $(a, \infty) \subset \rho(A)$ such that $R(\lambda, A) x=\int_{0}^{\infty} \lambda^{n} e^{-\lambda t} S(t) x d t$ for all $\lambda>a, x \in E$. This unique operator is called the generator of $(S(t))_{t \geq 0}$. Since we are mainly interested in generators, for $n \in \mathbf{N}$, a non-degenerate, exponentially bounded $n$-times integrated semigroup will be called an $n$-times integrated semigroup for simplicity.

It should be pointed out that for an $n$-times integrated semigroup $(S(t))_{t \geq 0}$ $(n \in \mathbf{N})$ with $\|S(t)\| \leq M e^{w t}$ for all $t \geq 0$, the constant $w$ must be non-negative. This follows from the equality

$$
S(t) x=\frac{t^{n}}{n !} x+\int_{0}^{t} S(s) A x d s,
$$

which holds for all $x \in \mathcal{D}(A)$ and $t \geq 0$. Similarly, if $(S(t))_{t \geq 0}$ is locally Lipschitz with $\|S(t+h)-S(t)\| \leq M e^{w(t+h)} h$ for all $t, h \geq 0$, the constant $w$ must be non-negative.

If $A$ generates an $n$-times integrated semigroup $(\widetilde{S}(t))_{t \geq 0}$, then for every $\lambda \in \mathbf{C}$, $A-\lambda$ generates an $n$-times integrated semigroup $(\widetilde{S}(t))_{t \geq 0}$, where

$\widetilde{S}(t) x=e^{-\lambda t} S(t) x+\sum_{k=1}^{n} \lambda^{k}\left(\begin{array}{l}n \\ k\end{array}\right) \int_{0}^{t} \int_{0}^{u_{k}} \cdots \int_{0}^{u_{2}} e^{-\lambda u_{1}} S\left(u_{1}\right) x d u_{1} \cdots d u_{k} \quad \forall x \in E$.

(To see this, it suffices to check that $\int_{0}^{\infty} e^{-\mu t} \widetilde{S}(t) x d t=\frac{1}{\mu^{n}} R(\mu, A-\lambda) x$.) The following two lemmas give the relation between the locally Lipschitz constants of $(S(t))_{t \geq 0}$ and $(\widetilde{S}(t))_{t \geq 0}$. 
Lemma 3.3. Let $n \in \mathbf{N}$. Suppose A generates an $n$-times integrated semigroup $(S(t))_{t \geq 0}$ satisfying

$$
\|S(t+h)-S(t)\| \leq M h \quad \forall t, h \geq 0,
$$

where $M$ is a constant. Then for every $\lambda>0, A+\lambda$ generates an $n$-times integrated semigroup $(\widetilde{S}(t))_{t \geq 0}$ with the property that given any $\epsilon>0$, there exists a constant $\widetilde{M}$ such that

$$
\|\widetilde{S}(t+h)-\widetilde{S}(t)\| \leq \widetilde{M} e^{(\lambda+\epsilon)(t+h)} h \quad \forall t, h \geq 0 .
$$

Proof. Let $\lambda, \epsilon>0$. Take $M_{1}>0$ such that $\|S(t)\| \leq M_{1} e^{\epsilon t}$ for all $t \geq 0$. Then for every $t, h \geq 0$, we have

$$
\begin{aligned}
& \|\widetilde{S}(t+h)-\widetilde{S}(t)\| \leq\left\|e^{\lambda(t+h)} S(t+h)-e^{\lambda t} S(t)\right\| \\
& \quad+\sum_{k=1}^{n} \lambda^{k}\left(\begin{array}{l}
n \\
k
\end{array}\right) \int_{t}^{t+h} \int_{0}^{u_{k}} \cdots \int_{0}^{u_{2}} e^{\lambda u_{1}}\left\|S\left(u_{1}\right)\right\| d u_{1} \cdots d u_{k} \\
& \leq e^{\lambda(t+h)}\|S(t+h)-S(t)\|+\left(e^{\lambda(t+h)}-e^{\lambda t}\right)\|S(t)\| \\
& \quad+\sum_{k=1}^{n} \lambda^{k}\left(\begin{array}{l}
n \\
k
\end{array}\right) \int_{t}^{t+h} \int_{0}^{u_{k}} \cdots \int_{0}^{u_{2}} M_{1} e^{(\lambda+\epsilon) u_{1}} d u_{1} \cdots d u_{k} \\
& \leq M e^{\lambda(t+h)} h+e^{\lambda(t+h)} \lambda h M_{1} e^{\epsilon t}+\sum_{k=1}^{n} \lambda^{k}\left(\begin{array}{l}
n \\
k
\end{array}\right) M_{1}(\lambda+\epsilon)^{1-k} e^{(\lambda+\epsilon)(t+h)} h \\
& \leq \\
& \quad\left[M+\lambda M_{1}+M_{1} \sum_{k=1}^{n} \lambda^{k}\left(\begin{array}{l}
n \\
k
\end{array}\right)(\lambda+\epsilon)^{1-k}\right] e^{(\lambda+\epsilon)(t+h)} h .
\end{aligned}
$$

Lemma 3.4. Let $n=1$ or 2 . Suppose A generates an $n$-times integrated semigroup $(S(t))_{t \geq 0}$ satisfying

$$
\|S(t+h)-S(t)\| \leq M e^{w(t+h)} h \quad \forall t, h \geq 0,
$$

where $M, w$ are constants. Then for every $\lambda>w, A-\lambda$ generates an $n$-times integrated semigroup $(\widetilde{S}(t))_{t \geq 0}$ satisfying

$$
\|\widetilde{S}(t+h)-\widetilde{S}(t)\| \leq \widetilde{M} h \quad \forall t, h \geq 0,
$$

where $\widetilde{M}$ is a constant.

Proof. Let $\lambda>w$. Take $\epsilon>0$ such that $\lambda>w+\epsilon$. It follows from the condition on $(S(t))_{t \geq 0}$ that there exists $M_{1}>0$ such that $\|S(t)\| \leq M_{1} e^{(w+\epsilon) t}$ for all $t \geq 0$. So for every $t, h \geq 0$, we have

$$
\begin{aligned}
& \|\widetilde{S}(t+h)-\widetilde{S}(t)\| \leq\left\|e^{-\lambda(t+h)} S(t+h)-e^{-\lambda t} S(t)\right\|+2 \lambda \int_{t}^{t+h} e^{-\lambda r}\|S(r)\| d r \\
& \quad+\lambda^{2} \int_{t}^{t+h} \int_{0}^{s} e^{-\lambda r}\|S(r)\| d r d s \\
& \leq e^{-\lambda(t+h)}\|S(t+h)-S(t)\|+\left|e^{-\lambda(t+h)}-e^{-\lambda t}\right| M_{1} e^{(w+\epsilon) t} \\
& \quad+2 \lambda \int_{t}^{t+h} M_{1} e^{(w+\epsilon-\lambda) r} d r+\lambda^{2} \int_{t}^{t+h} \int_{0}^{s} M_{1} e^{(w+\epsilon-\lambda) r} d r d s \\
& \leq e^{(w-\lambda)(t+h)} M h+\lambda e^{-\lambda t} h M_{1} e^{(w+\epsilon) t}+2 \lambda M_{1} h+\lambda^{2} M_{1}(\lambda-w-\epsilon)^{-1} h \\
& \leq \\
& \quad\left[M+3 \lambda M_{1}+\lambda^{2} M_{1}(\lambda-w-\epsilon)^{-1}\right] h .
\end{aligned}
$$


Lemma 3.5. Let $E$ be a Banach space and let $w, M \geq 0$. Suppose $F:[0, \infty) \longrightarrow E$ satisfies $\lim \sup _{h \rightarrow 0+} h^{-1}\|F(t+h)-F(t)\| \leq M e^{w t}$ for all $t \geq 0$. Then

$$
\|F(t+h)-F(t)\| \leq M e^{w(t+h)} h \quad \text { for all } t, h \geq 0 .
$$

Proof. It suffices to prove the result for the case where $E=\mathbf{R}$. First, we note that $F$ is Lipschitz continuous on every bounded interval in $[0, \infty)$. Indeed, for every $\eta>0$, take $M_{1}>M e^{w \eta}$; then we have $\limsup _{h \rightarrow 0+} h^{-1}|F(t+h)-F(t)|<M_{1}$ for all $t \in[0, \eta)$. From this it follows that $|F(t+h)-F(t)| \leq M_{1} h$ whenever $0 \leq t<t+h \leq \eta$.

Next, since $F$ is absolutely continuous on bounded intervals in $[0, \infty)$,

$$
\int_{0}^{t} F^{\prime}(s) d s=F(t)-F(0) \quad \text { for all } t \geq 0 .
$$

Hence for $t, h \geq 0$,

$$
|F(t+h)-F(t)|=\left|\int_{t}^{t+h} F^{\prime}(s) d s\right| \leq \int_{t}^{t+h} M e^{w s} d s \leq M e^{w(t+h)} h .
$$

Theorem 3.6. Let $E$ be a Banach space and let $A: \mathcal{D}(A) \subset E \longrightarrow E$ be a linear operator.

1. Let $n \in \mathbf{N} \cup\{0\}$. Suppose there exists $w \geq 0$ such that $(w, \infty) \subset \rho(A)$ and the function $F:(0, \infty) \longrightarrow \mathcal{B}(E)$ defined by

$$
F(\lambda)=\frac{1}{\lambda^{n}} R(w+\lambda, A)
$$

satisfies condition $\left(\mathrm{P}_{\infty}\right)$. Then A generates an $(n+1)$-times integrated semigroup $(S(t))_{t \geq 0}$ with the property that, given any $w_{1}>w$, there exists $M_{1}>0$ such that

$$
\limsup _{h \rightarrow 0+} h^{-1}\|S(t+h)-S(t)\| \leq M_{1} e^{w_{1} t} \quad \forall t \geq 0 .
$$

2. Let $n=0$ or 1 . Suppose A generates an $(n+1)$-times integrated semigroup $(S(t))_{t \geq 0}$ satisfying

$$
\limsup _{h \rightarrow 0+} h^{-1}\|S(t+h)-S(t)\| \leq M_{1} e^{w_{1} t} \quad \forall t \geq 0,
$$

where $M_{1}, w_{1}$ are constants. Then $\left(w_{1}, \infty\right) \subset \rho(A)$ and for every $w>w_{1}$, the function $F_{w}:(0, \infty) \longrightarrow \mathcal{B}(E)$ defined by

$$
F_{w}(\lambda)=\frac{1}{\lambda^{n}} R(w+\lambda, A)
$$

satisfies condition $\left(\mathrm{P}_{\infty}\right)$.

Proof. (1) By Theorem 2.3, there exists a constant $M>0$ and a function $T$ : $[0, \infty) \longrightarrow \mathcal{B}(E)$ satisfying $T(0)=0$ and $\|T(t+h)-T(t)\| \leq M h$ for all $t, h \geq 0$ such that for all $x \in E$,

$$
R(\lambda, A-w) x=\int_{0}^{\infty} \lambda^{n+1} e^{-\lambda t} T(t) x d t \quad \forall \lambda>0 .
$$

By [1, Theorem 3.1], $(T(t))_{t \geq 0}$ is an $(n+1)$-times integrated semigroup with generator $A-w$. Hence by Lemma 3.3, $A$ generates an $(n+1)$-times integrated semigroup $(S(t))_{t \geq 0}$ with the required property. 
(2) For every $w>w_{1}$, by Lemma 3.5 and Lemma 3.4, $A-w$ generates a Lipschitz continuous $(n+1)$-times integrated semigroup. Hence by Theorem $2.3, F_{w}$ satisfies condition $\left(\mathrm{P}_{\infty}\right)$.

Remark 3.2. The second assertion in the above theorem does not hold if $n \geq 2$. For example, in $\mathbf{R}$ or $\mathbf{C}, A=-1$ generates a 3-times integrated semigroup $(S(t)=$ $\left.-e^{-t}+\frac{t^{2}}{2}-t+1\right)_{t \geq 0}$ satisfying $\lim \sup _{h \rightarrow 0+} h^{-1}\|S(t+h)-S(t)\| \leq 2 e^{t}$ for all $t \geq 0$. However, $F_{w}(\lambda)=\frac{1}{\lambda^{2}(\lambda+w+1)}$ does not satisfy condition $\left(\mathrm{P}_{\infty}\right)$ for any $w$.

Corollary 3.7. Let $A: \mathcal{D}(A) \subset E \longrightarrow E$ be closed and densely defined and let $n \in \mathbf{N} \cup\{0\}$. If there exists $w>0$ such that $(w, \infty) \subset \rho(A)$ and the function $F:(0, \infty) \longrightarrow \mathcal{B}(E)$ defined by

$$
F(\lambda)=\frac{1}{\lambda^{n}} R(w+\lambda, A)
$$

satisfies condition $\left(\mathrm{P}_{\infty}\right)$, then $A$ generates an $n$-times integrated semigroup. The converse is true for $n=0,1$.

Proof. If $A$ satisfies the given condition, then by Theorem 3.6, $A$ generates a locally Lipschitz $(n+1)$-times integrated semigroup. Hence by [1, Corollary 4.2], $A$ generates an $n$-times integrated semigroup.

Conversely, for $n=0$ or 1 , if $A$ generates an $n$-times integrated semigroup $(S(t))_{t \geq 0}$ with $\|S(t)\| \leq M e^{w_{1} t}$ for all $t \geq 0$, where $M, w_{1}$ are constants, then $A$ generates an $(n+1)$-times integrated semigroup $\left(\widetilde{S}(t)=\int_{0}^{t} S(r) d r\right)_{t \geq 0}$ (in the strong operator topology) satisfying $\|\widetilde{S}(t+h)-\widetilde{S}(t)\| \leq M e^{w_{1}(t+h)} h$ for all $t, h \geq 0$. Hence the required result follows from Theorem 3.6.

To close our disscussion, we give the following example studied in [6].

Example 3.1. Let $E=L^{1}[0, R) \times L^{1}[0, R)$, where $R$ is a positive constant (larger than the life span of human beings). Let $A: \mathcal{D}(A) \subset E \longrightarrow E$ be given by

$$
A \varphi=\left(-\varphi_{1}^{\prime}-(\mu+\delta) \varphi_{1}+\sigma \varphi_{2},-\varphi_{2}^{\prime}-(\tilde{\mu}+\sigma) \varphi_{2}+\sigma \varphi_{1}\right)
$$

where $\mathcal{D}(A)$ consists of all $\varphi=\left(\varphi_{1}, \varphi_{2}\right) \in E$ with $\varphi_{1}, \varphi_{2}$ absolutely continuous and satisfying

$$
\begin{aligned}
& \varphi_{1}(0)=\beta \int_{0}^{R} h(r) k(r) \varphi_{1}(r) d r+\tilde{\beta} \int_{0}^{R} \tilde{h}(r) \tilde{k}(r) \varphi_{2}(r) d r \\
& \varphi_{2}(0)=\alpha \int_{0}^{R} h(r) k(r) \varphi_{1}(r) d r+\tilde{\alpha} \int_{0}^{R} \tilde{h}(r) \tilde{k}(r) \varphi_{2}(r) d r
\end{aligned}
$$

and $\mu, \tilde{\mu}, \sigma, \delta, k, \tilde{k}, h, \tilde{h}$ are nonnegative measurable functions on $[0, R)(\mu, \tilde{\mu}$ are the age specific mortality moduli of normal and disabled people; $0 \leq \sigma(r), \delta(r) \leq 1$ represent the recover rate and disabled rate at age $r ; 0<k(r), \tilde{k}(r)<1$ represent the proportion of the female population and that of the female disabled population of age $r ; h, \tilde{h}$ with $L^{1}$-norm equal to 1 are the birth modes of females and disabled females respectively) and $\alpha, \tilde{\alpha}, \beta, \tilde{\beta}$ are constants (which, in fact, depend on government population policy). Then $A$ satisfies the conditions given in Corollary 3.7 for $n=0$ (for details, see [6]) and thus generates a $C_{0}$-semigroup. 


\section{ACKNOWLEDGMENT}

The authors wish to thank the referee for his suggestions and comments on the original version of this article.

\section{REFERENCES}

[1] W. Arendt, Vector-valued Laplace Transforms and Cauchy Problems, Isreal J. Math. 59 (1987), 327-352. MR 89a:47064

[2] E.B. Davies, "One-parameter semigroups", Academic Press, London, 1980. MR 82i:47060

[3] E.B. Davies and M.M. Pang, The Cauchy problem and a generalization of the Hille-Yosida theorem, Proc. London Math. Soc. 55 (1987), 181-208. MR 88e:34100

[4] J. Diestel and J. J. Uhl, "Vector Measures", Amer. Math. Soc., Providence, 1977. MR 56: 12216

[5] R. deLaubenfels, Integrated semigroups, C-semigroups and abstract Cauchy problem, Semigroup Forum 41 (1990), 83-95. MR 91b:47092

[6] X. Li and J. Peng, Analysis of disabled population systems (Chinese), J. Xi'an Jiaotong Univ. 30 (1996) No. 10, 115-122. CMP 97:09

[7] D.V. Widder, "The Laplace transform", Princeton Univ. Press, Princeton, 1946. MR 3:232d

Department of Mathematics, Xi'an Jiaotong University, Xi'an 710049, People's RePUBLIC OF CHINA

Department of Mathematics, Hong Kong University, Hong Kong 